\title{
Aspectos para el desarrollo de una revista científica digital
}

\author{
Juan Voutssas M. *
}

Artículo recibido:

7 de octubre de 2011.

Artículo aceptado:

25 de julio de 2012.

\section{RESUMEN}

Este documento pretende analizar y establecer de forma integral y práctica todos los conceptos, aspectos y elementos que inciden en el desarrollo e implementación exitosa de una publicación periódica en formato digital -revista científica o e-journal-construida en función de los objetivos básicos de cualquier institución académica dedicada a la investigación y la docencia en el medio mexicano. Parte de esta investigación se estudió teóricamente en la literatura; otra parte se realizó observando una serie de características y atributos visibles en revistas digitales científicas existentes, y finalmente otra parte se desarrolló a través de la selección de una "institución tipo" donde se realizó un

* Instituto de Investigaciones Bibliotecológicas y de la Información de la UNAM, México.voutssas@unam.mx

INVESTIGACIÓN BIBLIOTECOLÓGICA, Vol. 26, Núm. 58, septiembre/diciembre, 2012, México, ISSN: 0187-358X. pp. 71-100 
estudio de caso analizando al interior de la misma el contexto, los recursos, las necesidades, etcétera. que ayudasen a establecer de forma integral todos los elementos que inciden en el desarrollo e implementación exitosa de un publicación Web de este tipo. Por el mismo contexto de este tipo de instituciones se contempla su desarrollo en un formato digital; esto es, bajo el concepto de e-journal, con o sin versión en papel. El estudio contempla los aspectos editoriales, tecnológicos, bibliotecológicos, administrativo-financieros y legales.

Palabras clave: Publicaciones digitales; Conceptualización, diseño y desarrollo de revistas científicas digitales; Aspectos editoriales, tecnológicos, legales, bibliotecológicos.

\section{ABSTRACT}

Consideration in the development of an online scientific journal

Juan Voutssas $M$.

This paper provides an analysis of the theory, functionality, nature and components of academic online journals known as -e-journals, e-zines, webzines, etc., as these are proposed under the typical goals pursued by a research institution in Mexico. The study focuses specifically on those facets involved in the initial development and launch of these publications. This research examines current literature in the field and assesses the features of existing online journals. Finally, a case study of an institution launching an online journal is offered, in which the context, needs and resources -including editorial, technical, bibliographic, management and legal requirements-- are thoroughly broken down for the purpose of identifying the key factors involved in the successful launch of a online journal.

Keywords: Digital publishing; Concept, design and development of academic e-journals; E-journals; Publishing, technical, legal, bibliographic, management issues; Web journals; Webzines. 


\section{INTRODUCCIÓN}

M uchas instituciones del medio mexicano dedicadas a la investigación y la docencia contemplan hoy en día la posibilidad de difundir sus trabajos y resultados mediante la creación de una publicación periódica en formato digital y en red: una revista científica o académica en soporte digital o "e-journal". En una gran mayoría de casos, este tipo de proyectos se ha realizado ya ajustándose a los paradigmas de una revista en soporte tradicional de papel, o bajo una óptica estrictamente computacional como si fuese un sitio Web más; estas visiones parciales inciden negativamente en el desarrollo y éxito de estas publicaciones. En realidad, la construcción de forma profesional de una revista científica digital conlleva un enfoque más holístico, una visión e integración de variados aspectos transdisciplinarios que influyen en su desarrollo adecuado y consecuente éxito: aspectos editoriales, tecnológicos, bibliotecológicos, administrativo-financieros y finalmente aspectos legales.

La correcta inclusión, balance e implementación de todos estos aspectos al momento de conceptualizar, diseñar y construir un e-journal en una institución académica maximizan su posible aceptación y éxito por parte de la comunidad académica usuaria, así como de los órganos certificadores. Para ello es pertinente establecer: cuáles son las características que definen a una publicación periódica ideal o e-journal para una cierta institución científica o académica; cuáles las diferentes opciones para construirla; cuáles son los atributos que buscan en los e-journals las diversas comunidades académicas que acceden a ellas; cuáles son los elementos requeridos por los repositorios bibliográficos y los organismos calificadores nacionales e internacionales que favorecen su ingreso a índices y listas de excelencia; cuáles son los aspectos de Tecnologías de Información y Comunicaciones -TIC- que deben cuidarse y, finalmente, cuáles son aquellos aspectos adicionales a los tecnológicos que deben ser contemplados: legales, económicos, etcétera.

Para contestar estas interrogantes se ha estudiado la literatura existente así como una serie de características y atributos relevantes y comunes en revistas existentes. Se seleccionó además una "institución tipo" para realizar un estudio de caso y observar al interior de la misma una serie de atributos, necesidades, contexto, etcétera, que ayuden a establecer y validar de forma práctica todos los elementos que inciden en el desarrollo e implementación exitosa de una publicación periódica en formato digital -revista científicaconstruida en función de los objetivos básicos de una institución académica dedicada a la investigación y la docencia en el medio mexicano.

En primer lugar es necesario establecer qué entendemos por revista "electrónica o "digital", ya que a pesar del tiempo que el término tiene ya de 
usarse y de lo extendido del mismo su acepción no es única ni clara, y es ahí donde empiezan los problemas. En la actualidad se utilizan ampliamente los términos en inglés: electronic journal, e-journal, e-magazine, e-zine, web-zine, e-newsletter, etcétera, así como sus acepciones en español: revista electrónica, revista digital, publicación periódica electrónica, boletín electrónico, etcétera. Además, dentro de esta vertiente electrónica se manejan las acepciones de "revista académica" o "revista científica", las cuales conviene precisar. Cabe hacer notar aquí que en este documento estas acepciones incluirán tanto a las publicaciones provenientes de las ciencias "duras" como a las de las ciencias sociales, las humanidades y las artes, y aunque nos referiremos a ellas en general como "revista científica", el término abarca esta visión más universal. Para entender bien estos conceptos, nomenclaturas y diferencias, es útil partir de su evolución.

\section{Antecedentes y ALCANCE}

A principios de la década de los ochenta, "publicación electrónica" se definía en general como toda aquella publicación -libro, revista, catálogo, bibliografía, etcétera- que era impresa con ayuda de una computadora sobre soportes tradicionales: papel, microformatos u otros. El término fue evolucionando en pocos años y derivando en libros electrónicos, revistas electrónicas, publicaciones Web, etcétera. Dada la naturaleza de este documento, aquí nos limitaremos específicamente al estudio de las publicaciones periódicas electrónicas o revistas electrónicas.

Los primeros antecedentes de estas "revistas" se remontan a principios de esa década: en 1980, la Universidad de Birmingham y la Universidad Tecnológica de Loughborough en Inglaterra, anunciaron conjuntamente el proyecto "BLEND" -Birmingham and Loughborough Electronic Network DevelopmentLos objetivos del proyecto eran estudiar los problemas de establecer una comunidad de información y elaborar una "revista electrónica" o "electronic journal”. El concepto de "revista electrónica” fue descrito en ese entonces como:

la utilización de las computadoras para ayudar los procedimientos normales por los cuales un artículo es escrito, arbitrado, aceptado y publicado. El autor, árbitros, editor y alternativamente los lectores pueden tener acceso al texto de los artículos así obtenidos desde sus computadoras.

En ese mismo año, Harry Collier presentó un documento en un taller de la Comisión de la Comunidad Europea acerca de la distribución de información 
científica y técnica con la ayuda de una computadora [Collier, 1981]. Con base en esa idea, para mediados de esa década la empresa "Learned Information" lanzó "The Electronic Magazine", una base de datos totalmente electrónica. Ésta estaba conformada por un conjunto de noticias técnicas y científicas de Europa y los Estados Unidos que eran reunidas en minicomputadoras y teletransferidas a un centro en Oxford, donde eran tratadas y combinadas con otras noticias y agregados recibidos de otros lugares, y se formaba así un "boletín" o "revista" electrónica con una selección de noticias, artículos cortos, revisiones de libros, publicidad, etcétera; es decir, un “e-magazine". ${ }^{1}$ Después de su proceso la revista era ofrecida para su consulta en-línea [Collier, 1984].

La Universidad de Siracuse, N.Y. publicó en 1987 a través de un proyecto especial la revista: "New Horizons in Adult Education", la cual fue la primera revista arbitrada distribuida vía la entonces existente red Bitnet, precursora de Internet; y que estaba en texto plano $A S C I I,{ }^{2}$ sin imágenes ni formato editorial y era gratuita. Todavía hoy puede verse en la red el primer número y observarse su precario formato [New Horizons..., 1987]. En 1989 apareció la publicación "Psycholoquy" (sic), una revista electrónica arbitrada de la American Psychological Association; en 1990 la siguió Postmodern Culture, otra revista arbitrada, también en ASCII a la usanza de la época y distribuida por la red, vía FTP -File Transfer Protocole-; todavía se produce hoy en día y está disponible en el sitio Web del proyecto "Musa". ${ }^{3}$ Si se revisa el estado del arte de estas revistas electrónicas, hacia 1990 puede observarse que no pasaban de una decena en el mundo [Okerson, Ann, 1991]. Éstas formaron lo que podríamos llamar las "protorevistas” electrónicas y tenían características comunes elementales: sólo texto plano ASCII, sin edición ni formato, sin imágenes ni otros multimedios, transmitidas antes de la Internet vía la incipiente red Bitnet, y transfería los contenidos de la revista vía un archivo anexo a un mensaje por medio del FTP. Éste es un protocolo normalizado que permitía de manera sencilla el intercambio de archivos de computadora entre equipos de distinta naturaleza, lo cual era toda una innovación en ese entonces. Ello daba la posibilidad de que las personas y organizaciones intercambiaran sus archivos con textos provenientes de su procesador de textos o sus bases de datos. No existía la "World Wide Web" ni el lenguaje html para publicación de textos. Muchos documentos fueron "publicados" así, como el simple intercambio de archivos

1 El "Magazine" es la revista de divulgación; es decir, aquella que contiene artículos, ensayos, revisiones, traducciones, editoriales, etcétera, dirigidos a una comunidad específica. De ahí se derivaron los términos: e-magazine, e-zine, web-zine.

2 ASCII: American Standard Code for Information Interchange o Código Americano Estándar para Intercambiar Información.

3 Disponible en: http://pmc.iath.virginia.edu/ 
electrónicos. Cabe subrayar que estas revistas iniciales eran primordialmente de origen universitario y que curiosamente las áreas predominantes de esas revistas eran las humanidades y las ciencias sociales. Esto se explica con el hecho de que en ese entonces el texto plano era el único que podía transmitirse y este tipo de temáticas se prestaba a ello, a diferencia de los artículos de ciencias "duras", que requieren en la mayoría de los casos de notación especializada, diagramas y otras ayudas visuales que en ese entonces era imposible publicar con estos medios. Las herramientas TeX, LaTeX, Postcript, Chiwriter, etcétera, propios para este tipo de documentos, se perfeccionarían hasta algunos años después.

Para la década de los noventa — la que podríamos llamar de la consolidación - se observan dos modalidades: primeramente, el advenimiento del CD-ROM en forma masiva y económica les permitió a los editores de publicaciones periódicas secundarias: índices, resúmenes, bancos de datos, bibliografías, catálogos, etcétera, distribuirlos ampliamente por este medio para ser usados en bibliotecas a través de sus relativamente recientes computadoras personales y "torres" lectoras de discos compactos. Un poco después aparecieron los $\mathrm{CD}$ ya con textos completos de publicaciones periódicas primarias. Esta primera modalidad tuvo gran impacto en la primera mitad de la década y fue cediendo su lugar gradualmente a una segunda variante que surgió a partir de 1994 con el auge de la red World Wide Web, el lenguaje html y los navegadores Mosaic y Netscape. Esta modalidad de publicaciones periódicas vía la Web ha seguido extendiéndose cada vez más hasta nuestros días siendo en la actualidad la más común por mucho. Para poder observar esta evolución, conviene basarnos en el "Directorio de Revistas Electrónicas, Boletines y Listas de Discusión Académica" -Directory of Electronic Journals, Newsletters and Academic Discussion Lists-, publicado por la "Asociación de Bibliotecas de Investigación" -Association of Research Libraries, o ARL-, el cual fue editado entre 1991 y 1997. Sigue disponible para consulta en la actualidad dentro del sitio "Web" de la ARL [Association of Research...., 2000]. En 1992 apareció la primera revista electrónica en texto completo, arbitrada y -lo más importante-incluía ya gráficas: El Online Journal of Current Clinical Trials, un esfuerzo de OCLC, -"Online Computer Library Center"- para apoyar el desarrollo de una revista por suscripción, electrónica y que no existiera también en papel. Fue un proyecto muy avanzado para su tiempo, ya que no existía la Web, ni los navegadores ni buscadores ni la edición html y requería por tanto de una interfaz especial para poder visualizarse.

La primera edición del directorio en 1991 consignaba 110 revistas y boletines electrónicos, así como 517 listas académicas de discusión. Para la tercera edición en 1993, las revistas eran 240 y las listas 1,152. Para 1994 se 
observan ya las primeras 11 revistas diseñadas específicamente para tomar ventaja de las características de la Web, y 60 eran distribuidas ya por este medio de un total de 443 revistas y boletines consignados; el número de listas y memorias de conferencias electrónicas ascendió a 1,758. En 1995, con la quinta edición del Directorio, 675 revistas y boletines eran consignados, si bien el número de revistas verdaderamente académicas era de 142. En el resto se notaba ya la potencialidad multimedial de la Web y múltiples "magazines" comenzaron a aparecer, dadas las capacidades de comercialización y divulgación de la "Web". Las memorias electrónicas de conferencias llegaron a 2,480, y se multiplicaron por diez con respecto a la primera edición. En la sexta edición en 1996, de un total de 1,689 revistas y boletines electrónicos consignados, más del 90 \% eran ya distribuidos vía la Web; sólo el 14 \% mantenía el correo electrónico como medio de distribución y el 17 \% ofrecía acceso por FTP. Las memorias y listas llegaron a 3,118. Para diciembre de 1997, con la última edición del directorio, la cuenta ascendía a 3,400 revistas y boletines, así como 3,800 memorias y listas. Del análisis de las ediciones del directorio es particularmente notable que hasta la tercera edición de 1993 ninguno de los títulos consignados en él poseía una dirección URL y para la sexta en 1996, más del $90 \%$ de los títulos ya la tenían; esto demuestra el impacto y crecimiento que en ese periodo tuvo la Web como promotora de la publicación electrónica, en especial las revistas científicas electrónicas.

Por lo mismo y para mediados de la década de los noventa, con el crecimiento de la red mundial WWW comenzó a darse por sentado para un gran sector de los usuarios que el término "publicación electrónica" era por antonomasia la "revista electrónica" y otros materiales semejantes que se publicaban vía la red, y empezaron a ser usados como sinónimos y a convertirse en el arquetipo de estas publicaciones. En mucha de la literatura del último lustro del siglo XX podemos observar este fenómeno y esta nomenclatura, pero en realidad debemos estar conscientes de que en realidad empezaban a utilizarse otras variedades de publicaciones electrónicas, si bien su desarrollo estaba más atrasado. La primera década de este siglo marca la "expansión" de las revistas científicas electrónicas. Para el cambio de milenio, la oficina del ISSN - International Standard Serials Number - consignaba aproximadamente 10,000 revistas electrónicas que se habían registrado en esa oficina.

Como puede verse, las primeras revistas electrónicas fueron simples copias de las versiones en papel trasladadas a soportes electrónicos casi a imagen; en pocos años éstas fueron evolucionando hacia modalidades más sofisticadas al incluir los multimedios y los servicios de valor agregado, lo cual empezó a diferenciarlas sensiblemente de las simples copias electrónicas de las revistas en papel, y les fue otorgando una "personalidad" propia conforme 
las publicaciones periódicas se hicieron más y más sofisticadas en la medida que la madurez intrínseca de los conceptos y la tecnología han ido evolucionando. Actualmente, las revistas electrónicas han adquirido características, especializaciones y facetas muy propios del medio electrónico e incluyen ya capacidades y variantes mucho más completas e inclusive inéditas con respecto a los soportes en papel .

A partir del cambio de milenio las revistas electrónicas fueron también cambiando su nomenclatura a "revistas digitales", como reflejo de esa madurez y de los conceptos introducidos a su vez por las "bibliotecas digitales"; por lo mismo, si bien teóricamente el concepto de "revista digital" presupone el uso de estos conceptos más avanzados con respecto a la original "revista electrónica", la realidad es que en la práctica hoy en día estos términos siguen siendo sinónimos. Obviamente el proceso evolutivo no está terminado y cada día pueden verse sutiles cambios que siguen conformando esa "personalidad propia" dentro de las revistas digitales. Dado que en la actualidad presentan múltiples variantes y características, hay por lo mismo varias formas de agruparlas [Kling y McKim,1997] distinguían ya hace poco más de una década cuatro tipos principales de revistas electrónicas o digitales:

- e-journals "puros": aquellos cuya publicación sólo se realiza en medio digital, sin mediar publicación previa en papel, como la Revista Digital Universitaria — RDU— de la UNAM o el Journal of Electronic Publisbing 一JEP.

- e-p-journals: aquellos que se distribuyen primordialmente en forma electrónica, pero de los que puede haber limitadas copias en papel. Por ejemplo The Journal of Artificial Intelligence Research y The Electronic Transactions on Artificial Intelligence.

- p-e-journals: aquellos que eran distribuidos primordialmente en papel pero ahora cuentan con versión digital. Como ejemplos tenemos $\mathrm{Na}$ ture, Investigación Bibliotecológica, Los Universitarios, Library Journal y Physical Review.

- p+e-journals: Aquellos que de inicio son lanzados con versiones en papel y electrónicas igualmente importantes. Como ejemplo está The American Chemical Society's Organic Letters.

En la última década se ha agregado una quinta categoría, denominada "blog". El término proviene de la contracción de un concepto de la red: "Web Log" —contenidos personales en la Red. Básicamente un "blog" es una revista electrónica con una serie de artículos que se distribuyen por la red WWW, con la característica distintiva de que su información es dinámica: se 
actualiza constantemente y en la mayoría de los casos contiene retroalimentación de los lectores. Si bien no es una modalidad muy utilizada en revistas científicas propiamente dichas, ha ganado mucha popularidad en revistas de difusión y divulgación científica y tecnológica y completa nuestra lista.

Por otra parte y de acuerdo con el tipo de acceso, las revistas pueden clasificarse entre las que son de "acceso pagado" por medio de una suscripción o compra de los artículos y las de "acceso abierto" — Open Access — que funcionan bajo un esquema de acceso libre para los usuarios. De estas últimas se observan las siguientes variedades [Melero, 2005]:

Las revistas que funcionan bajo algún esquema de suscripción y que después de un "embargo" de seis a doce meses facilitan el acceso abierto a sus contenidos o los depositan en algún repositorio abierto, como por ejemplo PubMed Central.

Las revistas de "acceso abierto" de paga, en donde los autores pagan por la publicación a la revista y retienen sus derechos de propiedad patrimonial de sus obras; por ejemplo: BioMed Central, Public Library of Science Biology.

Las revistas en las que la institución asume los costos de la publicación y exime a los autores del pago por publicar, a condición de que los materiales se publiquen y permanezcan en acceso abierto. Por ejemplo Investigación Bibliotecológica, Journal of Electronic Publishing.

Finalmente, existen algunas revistas "híbridas" que tienen una combinación de las dos anteriores, en donde la institución editora ofrece ambas variantes. Por ejemplo: Nucleic Acids Research.

Esta evolución a lo largo de tres décadas ha llevado a las revistas electrónicas hacia modalidades más sofisticadas que incluyen los multimedios y los servicios de valor agregado, lo que las diferencia sensiblemente de las simples versiones electrónicas de las revistas en papel y les va otorgando esa "personalidad" propia como una publicación periódica mucho más sofisticada en la medida que la madurez intrínseca de los conceptos propios y la Tecnología de Información y Comunicaciones van evolucionando. Actualmente las revistas electrónicas han adquirido características, especializaciones y comportamientos cada vez más propios del medio electrónico en la red. De acuerdo con todo lo anterior, si unimos las definiciones previas y se le agregan algunas precisiones finas, podemos aventurarnos a hacer una definición actualizada de revista científica digital:

Una serie editorial cuyo contenido está dirigido hacia una comunidad académica específica y es sujeto de arbitraje de sus contenidos por expertos; pretende aparecer indefinidamente en intervalos regulares, — con más frecuencia que anualmente-, 
y cada contenido es numerado o fechado consecutivamente en fascículos, aunque puede tener actualizaciones y comentarios; contiene normalmente materiales académicos originales, tales como artículos, ensayos, traducciones, reseñas, revisiones $\mathrm{u}$ otros escritos que han sido preparados para ser vistos en dispositivos electrónicos y puede o no tener equivalente impreso en papel. Se distribuye principalmente a través de redes de comunicación como la Internet, aunque también puede encontrarse en medios electrónicos portátiles como discos y memorias, y además de texto e ilustraciones, puede contener otras modalidades documentales, como audio y video, gráficas animadas, simulaciones, etcétera, así como servicios de valor agregado, como formatos alternativos, hipervínculos, diccionarios anexos, manejo de datos, foros de discusión, artículos con correcciones, etcétera.

Cabe resaltar en este punto los dos "perfiles" principales que una revista académica puede presentar: "journal" o "magazine". El primero tiene un corte más formal, y por lo general se limita a presentar artículos originales e inéditos resultados de investigaciones teóricas y aplicadas u otros productos de alto rigor académico. Contiene un resumen así como citas y referencias bibliográficas muy estrictas. Trata sólo sobre algún o algunos tópicos altamente especializados y sus materiales son escritos casi exclusivamente por y para investigadores o expertos, y son siempre arbitrados estrictamente antes de su publicación. El segundo tiene un panorama temático más amplio, y por lo general el concepto es asociado a una publicación informal dirigida a la divulgación entre un público en general. Una institución editora puede quedarse en este nivel, y es perfectamente válido diseñar una publicación de esta naturaleza. Existe también una variante del mismo: el e-magazine técnico-científico. Aquí el concepto puede ir más allá del concepto básico de este tipo de revista: consiste en una serie que no está dirigida a todo público en general sino a una audiencia de tipo profesional más amplia que la de los investigadores expertos pero que puede entender la temática y lenguaje de la publicación. Abarca por tanto otro tipo de modalidades de materiales que se publican, tales como traducciones, adaptaciones, reseñas, revisiones, reportes técnicos resultado de proyectos realizados; soluciones a problemas estudiados; ensayos; aplicaciones práctica, tales como mapas, cartas, tablas, diagramas; etcétera. Otra información útil para la elaboración de nuevos proyectos en la misma temática de la revista es el material relacionado y útil para la docencia, aplicaciones computacionales, etcétera; esta modalidad también conlleva arbitraje por expertos para garantizar el nivel de calidad de los contenidos. Finalmente, cabe mencionar que existen otras variantes de publicaciones periódicas digitales: boletines o "newsletters", gacetas, anuarios, etcétera, que son de menor uso y tienen otras características editoriales. En todo caso, 
si se desea producirlas, los principios básicos de este documento son válidos también para ellas en su inmensa mayoría.

Como ha podido observarse, existen varias maneras de agrupar para su análisis a las revistas digitales: por las variedades de su presentación, por el tipo o modalidad de la revista, por la temática, tipo de acceso y objetivos que la generan, etcétera. Como el objetivo de este documento es el de permitir comprender claramente una institución académica de investigación y docencia, la naturaleza y el perfil de una revista digital así como todos sus elementos básicos y complementarios de forma práctica para finalmente permitirle emprender la construcción rápida y eficiente de una publicación de esta naturaleza con un diseño profesional y holístico, se consideró conveniente dividir el proyecto en cinco apartados o aspectos que se deben desarrollar: aspectos editoriales, tecnológicos, bibliotecológicos, administrativo-financieros y finalmente aspectos legales.

\section{Aspectos editoriales}

Es de vital importancia que de inicio la institución editora reflexione profundamente en el objetivo de la revista digital que pretende desarrollar: debe establecer claramente cuál es el perfil de la revista que va a crear a partir de los objetivos que tiene la institución para con la revista diseñándola a partir de la comunidad objetivo a la que se pretende llegar y de la cual se extraerán en un futuro potenciales autores. Este es el aspecto que permite posteriormente definir las demás características de la revista en función del objetivo institucional que se persigue. En esta sección —que es crucial para el desarrollo de una revista - debe definirse su perfil, su conceptualización y diseño editorial; el tipo y corte de la revista; tipo y variedad de los lectores objetivo; el tipo, variedad y alcance de los materiales que publicará, y la periodicidad, indexación deseada, agencias evaluadoras objetivo, calidad, etcétera. Lo ideal es que el resultado del ejercicio en este aspecto se refleje en un documento de "política editorial" de la institución que marque la pauta para el desarrollo de la revista tanto en el presente como en el futuro.

Los elementos a considerar para el desarrollo de los aspectos editoriales son:

1) Definición por parte de la institución del tipo de revista digital que se va a producir entre los cuatro de la clasificación de Kling y McKim: ejournal "puro", e-p-journal, p-e-journal, $p+e$-journal.

2) Definición por parte de la institución del "perfil”, corte o sello de la revista: journal, magazine, newsletter o una mezcla de ellos. 
3) Detalle de las secciones que podrán existir en la revista. Aquí debe definirse si a las dos secciones mencionadas anteriormente -journal, magazine, newsletter- se desea agregarles otras secciones más: blog, foros de discusión, -moderados o no-, actualizaciones, sesiones de debate con los autores, etcétera. En todo caso cada una las secciones deberá estar siempre perfectamente diferenciada, delimitada y especificada en el sitio Web resultante.

4) Establecimiento, alcance, cobertura y límite de las temáticas que se incluirán en la revista.

5) Establecimiento de la frecuencia de aparición de la publicación y su nomenclatura — año, volumen, número- o como se desee establecer. Es recomendable que la publicación aparezca al menos dos veces al año para ser revista; si apareciera una sola vez al año se parecería más a un "anuario". Debe establecerse también el mínimo de artículos a editar por año; el mínimo anual aceptado generalmente son doce al año.

6) Establecimiento del tamaño máximo, mínimo e ideal de cada "fascículo"; determinación de si se cierran los fascículos a una fecha dada y no se agrega nada a la publicación hasta el nuevo fascículo — fascículo cerrado_ o si puede haber actualizaciones con "preliminares" — preprints - cada vez que se acepten nuevos artículos -fascículo abiertoEn ambos casos es fundamental que cada artículo quede asociado siempre a un fascículo y tenga una numeración correspondiente de forma unívoca; esto es, que cada artículo pertenezca a un y sólo un fascículo.

7) Esclarecimiento de la lista o listas con los criterios, requisitos y formatos que los posibles autores deben satisfacer al momento de someter trabajos a la revista y explicitarlas claramente.

8) Establecimiento de las características, perfil, funciones y número del grupo de pares evaluadores de los trabajos sometidos para cada sección de la revista. Debe hacerse una selección muy cuidadosa de expertos que garanticen un alto nivel de los contenidos de la revista.

9) Establecimiento del universo, origen y tipo de potenciales autores así como de los canales y posibilidades viables para la consecución de documentos. Esto en función de la posibilidad real de la organización para captar un cierto número de aportaciones en un periodo dado, ya que una vez establecido el tamaño mínimo del fascículo y la frecuencia será necesario respetarla escrupulosamente, como un mecanismo indispensable en un futuro cercano para ingreso y permanencia dentro de estándares de calidad y aceptación oficiales. Debe tenerse en 
cuenta que un criterio de calidad que será utilizado en las futuras evaluaciones será el porcentaje de autores internacionales que participen en la revista después de un tiempo.

10) Detección y establecimiento de las agencias revisoras y/o evaluadoras así como de las bases de datos del sector a las que se pretende acceder en un futuro con la revista, y de los requisitos emanados de las mismas que debe cumplir ésta.

Para establecer lo anterior puede usarse una metodología que contemple algún o algunos de los elementos enumerados a continuación:

1) La presentación de todos los diversos "tipos", "cortes" y "secciones" con todas sus diversas modalidades, opciones y variantes posibles en revistas digitales ante la comunidad de la institución, a todos los niveles de la misma: directivos, investigadores, técnicos, etcétera.

2) El recabado de visiones y puntos de vista de los diversos sectores de la institución acerca de sus preferencias y necesidades en lo respectivo a literatura científica acerca del tema.

3) El diseño y aplicación de una encuesta específica dentro de los diversos sectores de la institución y otras afines.

4) El estudio del perfil y las características técnicas, de contenido y presentación, de las revistas digitales académicas similares editadas actualmente. Establecer contra quién se compite o a quién se apunta como meta.

5) El estudio de los requisitos y condiciones establecidos por las principales organizaciones evaluadoras, calificadoras e indizadoras de las publicaciones digitales. Dependiendo del campo, nivel y temática de la revista, esta lista puede y debe variar, pero en términos generales puede partirse siempre de los requisitos del Conacyt (Criterios Generales de Evaluación Para el Índice de Revistas Científicas Mexicanas de Investigación Científica y Tecnológica del Consejo Nacional de Ciencia y Tecnología) ${ }^{4}$ los criterios del JCR (Journal Citation Reports) de Thomson-Reuters (antiguamente ISI), ${ }^{5}$ típicamente utilizados en revistas pertenecientes a las ciencias exactas y de la salud; los criterios de Latindex (Sistema Regional de Información para las Revistas Científicas de América Latina, el Caribe, España y Portugal), ${ }^{6}$ y los criterios de FECYT (56 Criterios de Calidad para la Evaluación de las

\footnotetext{
http://www.conacyt.gob.mx/Indice/Documents/CriteriosEvaliRICyT_v1.pdf http://isiwebofknowledge.com/media/pdf/Selection_essay-spanish.pdf

http://www.latindex.org/documentos/revistas_elec.html
} 
Revistas Científicas Españolas de la FECYT -Fundación Española Para la Ciencia y la Tecnología-). ${ }^{7}$ A éstos deberán agregarse los criterios inherentes a los organismos y revistas propios del sector y campo de conocimiento de la revista que se pretende construir, por ejemplo, LILACS para revistas en el sector de ciencias de la salud en Latinoamérica, el "International Bibliography of the Social Sciences" para ciencias sociales, "Library and Information Science Abstracts" -LISA- para bibliotecología, "Historical Abstracts" para historia, "Abstracts of Music Literature" para música, etcétera.

El objetivo es que la revista satisfaga desde su inicio los criterios y estándares establecidos nacional e internacionalmente, -en especial en su sector-, para su rápido ingreso a los índices de calidad. La institución debe considerar todos los estándares y requisitos aplicables durante su análisis preliminar, y luego decidir cuáles de ellos quiere satisfacer. Es recomendable establecer y tratar de cumplir los estándares que la organización desee desde el inicio de la revista. Una vez iniciada la misma es difícil después hacer cambios en sus propios estándares. Debe considerase también que existe un plazo de un año después del primer número para solicitar evaluación y ésta se da generalmente después de los dos años de este primer número.

6) El establecimiento de las características físicas de los materiales a recibir por sección: variedades, divisiones, formato, extensión, tipo de fuente de letra, interlineado, notas al pie, citas y referencias, anexos -diagramas, fotografías, audio, video, simulaciones, programas, etcétera y sus características. Los criterios de elegibilidad para cada trabajo a ser incluido dentro de cada sección pueden variar de una sección a otra, pero todos los trabajos en todas las secciones deben estar sujetos a arbitraje -y ser aceptados- antes de poder ser publicados. Los criterios deben estar perfectamente preestablecidos y ser accesibles entre los potenciales árbitros y autores.

7) Hay que establecer el perfil y número y nombres del cuerpo arbitral revisor pertinente a los criterios que éste deberá aplicar y los nombres de los expertos que conformarán ese grupo.

8) Es necesario establecer al interior de la institución un "Consejo Editorial" que se encargue de analizar, discutir y en su caso aprobar los diversos aspectos editoriales presentados previamente. Una vez aprobados es conveniente darles forma por medio de un documento de "Política Editorial" que una vez sancionado recoja todos los aspectos 
establecidos y sirva como marco para desarrollar la revista digital. Es muy recomendable que el Consejo Editorial continúe funcionando de tal forma que auxilie al titular de la institución y al editor de la revista en la determinación y perfeccionamiento de políticas y líneas de acción, supervise y que, en su caso, modifique la implementación y correcta operación de la revista digital. Para ello es conveniente preestablecer las características y funciones del "Consejo Editorial".

Los elementos que un documento de "Política Editorial" establece son en general:

a) Antecedentes y visión de la institución que crea la revista.

b) Definición, concepto, visión y temática de la revista digital.

c) Tipo, corte y secciones de la misma.

d) Criterios de elegibilidad de los trabajos sometidos: tipos, estructura, requisitos, etcétera.

e) Visión económica y financiera de la revista.

f) Conformación y funciones del Consejo Editorial.

g) Política legal de la aceptación y distribución de los materiales publicados.

9) Es conveniente también el establecimiento de las funciones del "editor" de la revista, encargado de gestionar todos los aspectos organizativos, administrativos, editoriales, etcétera, de la revista y nombrar una persona con esta función, ya sea como parte de la institución editora o como un consultor externo. Esta es una condición sumamente importante, ya que se requiere de un profesional especializado en esta función para velar por el cumplimiento de la política editorial, así como por la revisión, edición y publicación de cada artículo, entre muchas otras tareas. La falta de esta función y/o de un profesional especializado en ella conduce con frecuencia al incumplimiento de tiempos y niveles de calidad de la revista con su consecuente demérito y posterior desaparición.

\section{Aspectos TÉcNicos}

Se ha establecido que una característica típica de este tipo de revistas es su distribución y acceso sobre el servicio WWW montado sobre las redes de comunicación existentes, tales como lnternet, redes metropolitanas, redes Wan y más actualmente servicios GSM -Global System for Mobile Communicationspara dispositivos móviles: este hecho de ser construida sobre una estructura Web hace que frecuentemente se pretenda construir a la revista simplemente 
como un simple sitio Web más, basado en los paradigmas de construcción de estos sitios. Esto sólo es verdad en parte: en efecto, una revista construida como un sitio Web debe cumplir con una serie de principios y atributos técnicos propios de este tipo de instancias, pero sus objetivos, su imagen, su presentación, sus contenidos, etcétera, deben contemplar y reflejar en lo técnico los otros aspectos adicionales que pueden maximizar su éxito. La omisión de los demás aspectos reduce significativamente las probabilidades de éxito de la publicación. Aclarado este punto, en lo relativo exclusivamente a los aspectos tecnológicos a considerar en el desarrollo de una revista académica los principales puntos son:

1) Dimensionamiento de las capacidades tecnológicas de la institución: tipo y capacidad de servidores, redes, recursos humanos con los que cuenta, etcétera.

2) Dimensionamiento del tamaño de los acervos de la revista proyectados a un año, tres, cinco. Derivado de ello, contar con el espacio requerido de almacenamiento en disco.

3) Estimación de la demanda: "hits", descargas, ancho de banda, "podcast", etcétera, proyectados a uno y tres años. Derivado de estos dos puntos, determinar las características requeridas en el servidor donde se instalará la revista; y tener los programas necesarios dentro de él. Determinación del tipo y dimensiones de la red.

4) Establecimiento del entorno institucional de seguridad informática; esto es,

el proceso de establecer y observar un conjunto de estrategias, políticas, técnicas, reglas, guías, prácticas y procedimientos tendientes a prevenir, proteger y resguardar de daño, alteración o sustracción a los recursos informáticos de la organización y que administren el riesgo al garantizar en la mayor medida posible el correcto funcionamiento ininterrumpido de esos recursos.

5) Programas y aplicaciones necesarios para el desarrollo, edición, conversión y publicación de los documentos que van a publicarse.

6) Establecimiento del perfil y nivel de capacitación del personal técnico encargado de recibir, convertir, preparar y "subir" los documentos.

7) Establecimiento de programas y aplicaciones especiales que los lectores requerirán para consultar los diversos materiales: "flash", "visualizadores" especializados de imagen, sonido o video, etcétera, para poder informar a los usuarios de éstos. 
8) Establecimiento del o de los sistemas, metodologías y mecanismos de indización o "marcado" de los documentos que se van a publicar para su adecuado registro y recuperación dentro del catálogo y/o base de datos de la institución.

9) Determinación del "lay-out" o imagen esquemática de la revista y su "mapa del sitio".

10) Costo y proyección de estos aspectos tecnológicos, ya que este aspecto es uno de los que más incidirá sobre los costos de la revista.

11) Adopción de los estándares técnicos de construcción mínimos y obligatorios del sitio Web de la revista, los cuales pueden variar dependiendo del resultado de estudio realizado dentro de los aspectos editoriales acerca de los requisitos y condiciones establecidos por las principales organizaciones evaluadoras, calificadoras e indizadoras de las publicaciones digitales. Una visión completa de estos estándares es la siguiente:

a) La revista debe contar con su propio $\mathrm{URL}^{8}$ independiente del utilizado por la institución; es decir, no debe ser un simple subconjunto - "slash" o "dos puntos" - del sitio principal de la institución. Este URL debe aparecer explícitamente en todas las páginas Web de la revista, incluyendo las de los artículos y otros materiales, para oportuna identificación, citación y referencia.

a) La construcción de la página principal y subpáginas del sitio Web de la revista debe cumplir con las especificaciones y

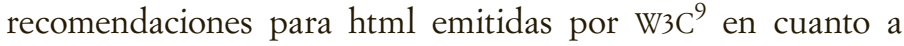
usabilidad, y los tipos de fuentes, colores, distribución física, navegación, etcétera [W3C, 2011]. Sobre todo debe funcionar compatiblemente con la mayoría de los navegadores existentes (explorer, firefox, opera, safari, chrome, etcétera) hasta una versión anterior a la actual. Por ningún motivo debe quedar atada a un navegador en específico ni ostentar ninguna leyenda de "esta página se ve mejor con..."

En lo relativo a la "presentación" o vista, en términos generales es recomendable que la revista cumpla con los establecido con el estándar ISO 8: 1977. Presentation of periodicals —Presentación

8 URL o "Universal Resource Locator" - "Localizador Uniforme de Recursos"; se refiere a la dirección única que identifica a una página dentro de la WWW, como por ejemplo http://www. unam.mx

9 W3C o "World Wide Web Consortium, es un consorcio internacional que produce recomendaciones y estándares para la WWW, en especial en lo tocante a URL's, el protocolo http y el lenguaje html. 
de las publicaciones Periódicas. ${ }^{10}$ Este estándar fue establecido para publicaciones en papel, pero los elementos requeridos en la presentación han cambiado poco y pueden ajustarse con facilidad a las publicaciones digitales. El punto "3", a continuación, es un desarrollo práctico que cumple con este estándar.

c) La revista debe mostrar claramente en su página principal una serie de "zonas" o secciones de información específica claramente visibles y diferenciables; estas "zonas" son:

- Zona de encabezado- La página Web de la revista debe contener los siguientes elementos: escudo o logo oficial y nombre completo de la institución y en su caso su acrónimo, más la leyenda "México"; nombre completo de la revista; y en su caso, nombre mnemónico de la revista.

- Zona del "Mapa del sitio".

- Zona de "derechos de autor" o "Copyright" con hipervínculo a un recuadro que informe acerca de las condiciones de licenciamiento y uso legal de los materiales de la revista. Si se opta por un licenciamiento tipo "Commons", el logotipo correspondiente de esta licencia debe estar visible en esta zona de la página.

- Recuadro resumen de la "página descriptiva (portada)" en la página principal del sitio de la revista, se consigna: el nombre oficial de la revista, el ISSN, el nombre de la institución como editor, frecuencia, objetivo y cobertura temática. Este recuadro debe tener un hipervínculo a una "Página Descriptiva" o "Portada" completa, en donde se deben consignar claramente:

- El nombre oficial de la revista, el ISSN, la frecuencia y fechas de aparición, el nombre completo de la organización como editor más la leyenda "México"; su dirección postal completa; el nombre, cargo y correo electrónico del director y/o editor de la revista; los títulos y nombres de los miembros del Consejo Editorial, sus instituciones y dependencias de adscripción o proveniencia claramente detalladas; los títulos y nombres detail.htm?csnumber $=3585$ 
del cuerpo arbitral completo con sus instituciones y dependencias de adscripción (sin correos electrónicos, ni dirección postal, ni teléfono); correo electrónico oficial de la revista; sitios donde la revista está indizada (directorios, resúmenes, índices, bases de datos, etc., ya sean electrónicos o no). La descripción de la revista en forma breve, objetivo, alcance y cobertura temática; información acerca de todos los registros obtenidos ante organizaciones indizadoras o de certificación, o al menos datos del inicio de su registro; la portada debe estar traducida al inglés para ser conmutada con el botón de idioma.

- Zona, línea o recuadro del "número actual" que incluye: nombre mnemónico de la revista; ISSN; fecha de aparición; año, volumen y número; índice o tabla de contenido de los artículos de la revista con sus autores, divididos por secciones independientes: "journal", "magazine" y "otras"; cada línea de cada artículo de la revista estará hipervinculada con el resumen bilingüe del artículo, -de existir- y éste a su vez con el artículo en texto completo; una lista del total de los números disponibles con hipervínculos a cada uno de ellos.

- Zona, línea o recuadro de "números anteriores" con un hipervínculo a otra subpágina con la información de todos y cada uno de los números publicados anteriormente, que incluya: fecha de aparición; año, volumen y número.

- Zona, línea o recuadro de "si deseas someter una obra a la revista" con hipervínculo visible a las "instrucciones para autores".

- Zona, línea o recuadro con hipervínculo visible a las "instrucciones para árbitros".

- Una "zona de colofón” en la parte inferior de la página con los siguientes elementos:

- La leyenda: "Ciertos Derechos Reservados Sobre el Sitio Web" a nombre de la institución en inglés y español.

- Botón de "créditos" de la página Web y de la revista, la cual a su vez contiene dentro de ella una 
zona de "créditos históricos" para poder actualizar a los responsables a lo largo del tiempo.

- Botón de "contacto" especificando que se trata de contacto para aclaraciones y dudas sobre el sitio. Las aportaciones de artículos van en la zona de "contribuciones a la revista" que no forma parte del colofón. Debe consignarse el correo electrónico y teléfono del responsable del contacto del sitio.

- Fecha de última actualización del sitio Web.

- Directorio de la revista.

- Estadísticas.

d) La totalidad de los trabajos publicados en los números anteriores de la revista debe quedar accesible de forma acumulada, con algún mecanismo de acceso y/o búsqueda de esos materiales.

e) La página y todas las subpáginas de la revista deben tener un hipervínculo visible al sitio Web principal de la institución. Todas las subpáginas deben tener un botón muy claro de "regreso a página anterior" y otro de "inicio" -home-. Debe evitarse en lo posible la necesidad de utilizar la barra de herramientas del navegador.

f) El Sitio Web de la revista de la institución debe tener una serie de especificaciones generales de diseño gráfico de entre las aceptadas en general por la comunidad usuaria de estos sitios; como mínimo:

- Debe contar con una identidad gráfica que transmita el sentido de pertenencia institucional a la organización que la crea.

- La imagen gráfica del sitio de la revista debe contar con una resolución ideal de 1024 x 768 pixeles con un mínimo de $800 \times 600$. Esta imagen gráfica seleccionada para el sitio no debe afectar su funcionamiento, no debe ser de peso excesivo y debe conservar tiempos promedio de carga y recarga razonables. El contenido gráfico no debe obstaculizar el posicionamiento del sitio Web; antes bien debe facilitar su rastreo e incorporación a bases de datos y buscadores.

- La presentación de contenidos en cada página debe respetar la estructura usual jerárquica de izquierda a derecha, arriba a abajo. 
- El contraste de la letra y su relación color-tamaño debe permitir leer todos los textos, inclusive de los letreros más pequeños, aun en pantallas pequeñas tipo netbook $\mathrm{y}$ teniendo en mente a personas con capacidades diferentes.

- Los colores de fondo siempre deben ser claros y las letras en colores oscuros para maximizar la legibilidad y facilitar la impresión.

g) En su caso, la revista puede desear construir una serie de elementos de valor añadido tales como:

- Buscador de artículos dentro de la revista (autor, título, palabras clave, etcétera).

- Servicio de "Alerta" de nuevos materiales recién aparecidos en las diversas secciones.

- Conmutación de todas las páginas de la revista en sus textos propios -no en los contenidos de los artículos- al idioma inglés y en su caso, a otros adicionales.

- Servicio de "Diseminación Selectiva de Información" donde se construya una base de personas interesadas en ciertos temas y se envíen materiales nuevos a los correos de esas personas ("push information services").

- Servicio de enlaces sindicados de fuentes Web codificadas en XML (RSS).

- Foros de discusión moderados.

- Materiales descargables por podcast y/o webcast.

- Versión "ligera" del sitio Web de la revista para dispositivos portátiles manuales: netbooks, pads, teléfonos, etcétera, [Mobile...., 2006].

\section{Aspectos BibLiográficos}

El desarrollo de estos aspectos tiene que ver con la necesidad de asegurar la calidad y homogeneidad de los contenidos desde un punto de vista bibliotecológico, estandarizando en lo posible la estructura de los registros al tiempo que se facilita su búsqueda, recuperación e inserción dentro de otros mecanismos localizadores dentro de la Web más allá del ámbito cercano de la revista. Dentro de los "aspectos editoriales" ya hemos mencionado una serie de elementos de calidad que también tienen que ver con los aspectos bibliotecológicos: tramitación de ISSN, desarrollo de una portada o membrete estandarizado, etcétera. Es pertinente en 
este punto agregar además el desarrollo de elementos bibliotecológicos. Para establecer lo anterior puede usarse una metodología que contemple algún o algunos de los elementos enumerados a continuación:

1) La revista debe cumplir con los estándares de calidad y presentación estipulados ya en el apartado de aspectos editoriales, resultado del estudio de los requisitos y condiciones establecidos por los principales repositorios bibliográficos así como de las organizaciones evaluadoras, calificadoras e indizadoras de las publicaciones digitales: ISSN, periodicidad escrupulosa, seriación -año, volumen, número-, contenido y colofón; etcétera.

2) Todos y cada uno de los artículos o materiales publicados debe tener su conjunto de metaetiquetas en alguna especificación preestablecida por la institución editora. Entre más rico o sofisticado sea el conjunto de metaetiquetas, más facilitará su hallazgo después por los buscadores de la Web, condición que adquiere suma importancia ya que la revista se distribuye por esta vía. En la práctica se observa el uso de los estándares METS - The Metadata Encoding and Transmission Specification - y MODS - The Metadata Object Description Scheme - como sistemas de manejo de metadatos construidos sobre una tradición MARC [Dappert, 2008]. El conjunto mínimo recomendado de metadatos es el de Dublin Core [Apps, 2000]. ${ }^{11}$ Cada vez más se observa el uso de estos estándares dentro de los e-journals bajo codificación XML. Independientemente del sistema que vaya a ser usado, el punto vital aquí es que las metaetiquetas asignadas a cada artículo se vean reflejadas en los "headers" de la versión html del documento para que puedan ser recuperadas con más precisión y facilidad por los buscadores de la red y así maximizar la accesibilidad y distribución de esos artículos dentro de la Web.

3) Cada artículo o material publicado debe tener un abstract o resumen y un conjunto de palabras clave tanto en inglés como en español.

4) Las citas y/o referencias bibliográficas deben ser incluidas en cada artículo bajo una estricta estandarización que permita en un futuro agruparlas e hipervincularlas; se recomienda el uso del estándar ISO 690-1:1987 —documentos impresos- e ISO 690-2:1997 —documentos electrónicos-, o uno semejante.

5) Para facilitar su descarga, lectura, citación y reuso, cada artículo debe 
estar accesible al lector en más de una versión; se recomienda usar formatos html, pdf descargable y versión electrónica de "hojas plegables" o "flip page". ${ }^{12}$ En el caso de que así se haya establecido previamente, deberán existir además las versiones "ligeras" de los artículos para dispositivos móviles: "netbooks", tabletas, celulares, etcétera.

Cada artículo o material publicado debe contar siempre con un "membrete bibliográfico" apropiado y estandarizado; esto es, consignar siempre en su primera página y de una forma totalmente normalizada, todos los elementos bibliográficos del mismo: además del nombre de la revista, el o los nombres de los autores, su institución y dependencia de adscripción, su correo electrónico profesional, las fechas de sometimiento a arbitraje, de aceptación y de publicación del documento, así como el título del artículo o material y en su caso, sus resúmenes bilingües. Además del membrete de la página principal del artículo, en todas y cada una de las páginas adicionales de los artículos deben repetirse de manera discreta en un pie de página o encabezado el nombre de la revista, su URL, el nombre de los autores, fecha de publicación y título del artículo para oportuna identificación, citación y referencia. Este elemento refuerza además los aspectos legales si consigna claramente en cada página de cada artículo la propiedad intelectual de la institución editora.

6) Es recomendable que la institución editora estudie la conveniencia de adoptar el número DOI ${ }^{13}$ "Identificador Digital de un Objeto" para cada artículo; si decide adoptarlo, éste debe ser consignado en el membrete y en cada página adicional del artículo.

\section{Aspectos Legales}

Dado que se trata de una revista que distribuye sus contenidos de forma digital y remota, estos aspectos son de suma importancia para la institución editora, ya que le permiten asegurar la propiedad intelectual de los materiales que edita pero buscando al mismo tiempo maximizar su alcance y distribución.

12 Se define como "hoja plegable" o "flip page" a las aplicaciones computacionales que simulan que una imagen digital de una página de un libro, revista etc. sea "hojeada" o pasada de un lado a otro como si fuera la página de un libro en papel. http://www.flashpageflip.com

13 El número DOI - Digital Object Identifier - es un sistema parecido a los identificadores ISBN e ISSN, pero creado para cada documento en particular. Se obtiene una membresía por parte de la International DOI Foundation; se envía y se registra cada artículo o material científico en el sitio al efecto y se recibe un número DOI para anexar a este material. A diferencia del sistema URL, el sistema DOI no cambia con el paso del tiempo, aunque el artículo sea reubicado en una dirección distinta puesto que lleva la información incorporada en forma de metadatos. Puede considerarse un URL permanente. http://www.doi.org 
Estos dos elementos son de suma importancia y rigen prácticamente todo el desarrollo de los aspectos legales. Por un lado la revista debe asegurarse que posee efectiva y cabalmente los derechos patrimoniales -esto es, de explotación- de cada contenido que distribuye con objeto de evitar futuras reclamaciones y disputas debidas a lagunas o acuerdos tácitos. Por otro lado es lógico desear la máxima penetración de la revista, y por ello debe diseñarse un fácil y amplio acceso, uso y distribución de sus materiales dentro de la comunidad académica a la cual se dirige la publicación. Para esto, la institución debe comenzar con la determinación exacta de las características legales con las que adquiere las colaboraciones y guardar documentación escrita de lo anterior, así como establecer y explicitar muy claramente las condiciones de reproducibilidad de los documentos publicados en la revista; esto es, cuál es el tipo de licencia que está otorgando a los lectores de la misma con respecto a los contenidos; y cuáles son las atribuciones y límites otorgados al lector y las condiciones en las que él puede acceder, descargar, usar, modificar, etcétera, esos materiales, tanto para ambientes comerciales como académicos, ya que es naturalmente deseable que la revista y cada artículo utilizados en otras obras sea citado adecuadamente

Para establecer lo anterior puede usarse una metodología que contemple los elementos enumerados a continuación:

1) Con objeto de asegurar la propiedad y unicidad del nombre de la revista es necesario crear una "Reserva de Derechos de Uso Exclusivo". El artículo 173 de la Ley Federal del Derecho de Autor señala que la propiedad consiste en la facultad de usar y explotar en forma exclusiva títulos, nombres, denominaciones, características físicas y psicológicas distintivas, o características de operación originales aplicadas de acuerdo con su naturaleza. Esto debe hacerse con el nombre oficial y estándar de la revista — nombre largo- y en su caso el nombre mnemónico o corto ante el Instituto Nacional del Derecho de Autor - INDAUTOR. Una vez hecho este registro puede solicitarse el ISSN el cual será otorgado al aparecer el primer número de la revista. Debe tramitarse además el nombre de dominio del sitio Web exclusivo de la revista ante NIC México o algún representante. En su caso, si se involucran en la revista logos o marcas deberá hacerse el registro correspondiente ante el IMPI, - Instituto Mexicano de la Propiedad Industrial—; todo lo anterior con objeto de tener asegurados todos los registros de ley de nombres y dominios.

Es sumamente importante no omitir el registro del ISSN. Ninguna revista que se respete y que pretenda entrar a los sistemas de indización 
internacional carece de este número; además permite identificar una revista de forma inequívoca, sustituye los largos registros de descripción bibliográfica, y evita errores al copiar referencias o documentos.

2) Determinación y establecimiento de los mecanismos que le aseguren a la institución editora la propiedad patrimonial permanente de los documentos publicados por la revista: cartas de cesión de derechos patrimoniales por parte de los autores, en los que se establece unicidad o multiplicidad de uso y versiones, exclusividad, compromisos de documento inédito previamente, originalidad de la publicación, titularidad de los derechos como autor o autores primigenios, etcétera; tales cartas deben elaborarse en todos los idiomas en los que se acepten materiales documentales por parte de la revista y obtenerse sin excepción por parte de todos los autores previamente a la publicación. Esto asegura la propiedad patrimonial de la revista al tiempo que la deslinda de futuras reclamaciones autorales.

3) Determinación y establecimiento de las condiciones de reproducibilidad de los documentos publicados en la revista por parte de los lectores: licencias, citas, modificaciones, atribuciones, colaboraciones, obras derivadas, etcétera, para ambientes académicos y/o comerciales. En su caso, determinación de los costos de estos licenciamientos. En el caso de revistas científicas o académicas que deciden no trabajar bajo el esquema de "todos los derechos reservados”, lo más común es operar y licenciar bajo el esquema de "ciertos derechos reservados": esquemas de licenciamiento "Open Access" tipo GNU —General Public License o GPL_, o el más utilizado de todos estos en la actualidad, la estructura conocida como "commons". Se deben analizar y seleccionar los atributos deseados de la revista bajo este régimen, registrarlos en su "Política Editorial" y hacerlos claramente explícitos a sus lectores y potenciales autores; esto se realiza consignando en el texto de la página Web de la revista la declaración de "commons" propia de esa revista. En términos generales, el conjunto de atributos más comúnmente seleccionados para este tipo de revistas es el denominado "cc by-nc-sa”. Attribution Non-Comercial Share Alike 2.5 Unported, o (Reconocimiento, No-Comercial, Compartir bajo la misma licencia - versión 2.5. El detalle completo de este tipo de licencia de "creative commons" puede verse en: http://creativecommons.org/licenses/by-nc-sa/2.5/mx/legalcode 
El aspecto administrativo tiene que ver con los elementos que la institución editora debe planear y ejecutar para la difusión y penetración de la revista antes y después de ser lanzada con objeto de lograr su posicionamiento en el medio. Tiene que ver también con el acopio y análisis de información que le permita a la institución editora retroalimentar los resultados y tendencias de usuarios, autores, etcétera, hacia acciones que corrijan posibles debilidades y potencien las fortalezas de la revista. El aspecto financiero requiere de un plan cuidadoso a corto y mediano plazo. Ya se ha establecido que entre los principales problemas que enfrentan las revistas científicas se encuentra la alta "mortalidad prematura" y la frecuente falta de regularidad [Cetto, 1998]. Estos problemas provienen principalmente de dos causas: la falta de contenidos documentales de buen nivel para la revista, lo cual tiene que ver con su posicionamiento, aceptación y calidad, y en segundo lugar la falta de financiamiento de la institución editora.

Para establecer lo anterior puede usarse una metodología que contemple los elementos enumerados a continuación:

1) Estrategia de penetración y "mercadeo" de la revista. Por lo general esto se basa en dos líneas de acción: en primera instancia se busca lo más rápidamente posible la acreditación de la revista ante la comunidad lectora; esto se puede lograr desde el inicio produciendo contenidos de alta calidad e interés para esa comunidad. Dado que los autores académicos y/o científicos desean obtener la máxima visibilidad para sus trabajos, tratarán por tanto de publicar preferentemente en las revistas con mayor penetración y difusión. En la medida que la calidad y penetración de la revista crece, más autores de importancia se van obteniendo, y la reputación y visibilidad se ven así incrementadas cada vez más, y se va creando un círculo virtuoso. Por supuesto, esa alta calidad debe ser mantenida a lo largo del tiempo, situación que no siempre es fácil de lograr; si la calidad decrece y se pierde credibilidad en la revista, el círculo se irá convirtiendo en vicioso. En segunda instancia -y a mediano plazo- se busca la inclusión dentro del mayor número posible de los repertorios bibliográficos y bases de datos del medio así como la acreditación y reconocimiento ante las agencias certificadoras e indizadoras de estas publicaciones, en especial las relacionadas con el sector al que pertenece la revista; esto se logra observando escrupulosamente los requisitos establecidos por ellas y que fueron incluidos en el diseño de la revista. 
En el caso de que la revista funcione bajo el concepto de "acceso abierto” es conveniente, -una vez teniendo la revista establecida y en operación- consignar el sitio y sus características dentro de los sitios mundiales que registran e indizan a las revistas de este tipo, como por ejemplo el "DOAJ - Directory of Open-Access Journals", ${ }^{4}$ "Open JGate”, etcétera. Esto con el fin de impulsar su penetración y visibilidad.

2) Establecer las bases de los mecanismos que se usarán para detectar las características y perfil de los lectores: encuestas en el sitio Web, cuentas de acceso, descargas, regresos, dominios desde los que se accede, etcétera. Es importante empezar a establecer claramente desde el principio de la revista los perfiles de los usuarios de la manera más detallada posible y establecer los contadores pertinentes para ir recabando la información. Como es ya sabido, la simple cuenta de accesos — "hits" - al sitio no basta.

3) Establecer el perfil del encargado de diseñar, aplicar e interpretar estos sondeos con fines de retroalimentación a la revista. El Consejo Editorial debe establecer y supervisar planes y medidas de retroalimentación con los resultados así obtenidos para incrementar la calidad de la revista y así corregir eventuales fallas y omisiones y potenciar las fortalezas y aciertos.

4) Establecer un plan de viabilidad y alternativas financieras de la revista. Establecimiento de materiales y/o versiones susceptibles de cobrarse así como sus posibles valores y destinatarios. Establecimiento de la posibilidad de que exista publicidad pagada, y -en este caso-, tipo, alcance y condiciones de la misma.

5) Establecer el plan de mantenimiento y renovación de la infraestructura tecnológica relativa a la revista.

\section{Conclusiones}

Como ha podido observarse, el diseño e implementación de una revista científica digital no puede hacerse simplemente ajustándose a los principios que rigen la creación de una revista en soporte tradicional de papel, ni bajo una óptica estrictamente computacional como si se tratase del desarrollo de un sitio Web más; estas visiones parciales afectan sensiblemente y de forma negativa las características de la revista, reduciendo sus probabilidades de éxito y prevalencia. Como se ha establecido, la construcción profesional de una 
revista científica digital conlleva un enfoque más holístico, una visión de variados aspectos transdisciplinarios que influyen en su desarrollo adecuado y consecuente éxito. Hemos agrupado y desarrollado estos factores en: aspectos editoriales, tecnológicos, bibliotecológicos, administrativo-financieros y finalmente aspectos legales. El correcto análisis, ponderación, inclusión, balance e implementación de todos estos aspectos al momento de conceptualizar, diseñar y construir un e-journal en una institución académica maximizan su posible aceptación y éxito por parte de la comunidad académica correspondiente, así como la de los órganos certificadores.

Las revistas científicas digitales fueron menospreciadas por muchos años en los países en desarrollo, cuando eran precisamente estos países quienes más podrían haberse beneficiado de su uso y aceptación. De acuerdo con la historia del directorio de ARL, en los noventas fueron mayormente las universidades e institutos de investigación quienes hicieron detonar este nuevo medio prácticamente de la nada a más de diez millares en poco más de una década; las instituciones académicas probaron entonces sobradamente que este medio podía y debía usarse como un gran vehículo de comunicación de la ciencia. No obstante, no fue sino hasta que las grandes editoriales "afamadas" convirtieron sus versiones en papel de sus revistas y comenzaron a venderlas a las instituciones académicas que estas empezaron a darles el valor y la importancia que hoy tienen. Gracias a los movimientos de "Open Access", "Commons", "Repositorios Institucionales", etcétera, un número cada vez mayor de instituciones académicas ha comenzado nuevamente a contemplar la salida de sus resultados y productos académicos a través de la construcción de revistas académicas digitales. Muchas de las instituciones académicas mexicanas dedicadas a la investigación, a la docencia y a la innovación tecnológica están también en capacidad de aprovechar su infraestructura tecnológica y sus recursos humanos de alto nivel y especialidad dotándose de una herramienta, un -e-journal-, que impulse su quehacer para el avance de la investigación, la docencia, la vinculación y la divulgación en México, dándole sinergia al proceso de generación de conocimientos y desarrollos tecnológicos originales y de punta, y ampliando y fortaleciendo su posicionamiento en el ámbito científico y tecnológico. Igualmente, una revista así articula los procesos de generación y transferencia de conocimiento e innovaciones con las necesidades de sus usuarios, socios académicos y/o clientes permitiéndoles insertarse en su contexto en óptimas condiciones para intercambiar información, conocimiento y habilidades con otras organizaciones académicas, de gobierno y de la sociedad civil que requieren de sus resultados y conocimientos para la solución de problemas.

Al margen de los problemas derivados de políticas de publicación y de la voluntad de realizar estos proyectos es necesario emprenderlos de una manera 
profesional, integral y planeada, de tal forma que las probabilidades de éxito se maximicen; no hacerlo así pone en riesgo la viabilidad del proyecto. $\mathrm{Mu}-$ chas de las revistas desaparecen al cabo de pocos números debido a esta falta de planeación e integración de los aspectos críticos que inciden en la producción de una revista de esta naturaleza: la carencia de personal editorial y técnico especializado profesionalmente en el tema, de un plan integral de desarrollo y deficiente financiamiento las lleva con frecuencia a una falta de regularidad, pobre producción y baja aceptación de la comunidad objetivo y de las agencias que las certifican y consignan, y estas revistas terminan desembocando en un proyecto fallido.

Obviamente no existe una receta mágica que garantice cien por ciento el éxito en un proyecto de esta naturaleza, pero sin duda el desarrollo de un proyecto elaborado en las mejores condiciones que maximicen sus probabilidades de éxito es una mejor alternativa.

"Nunca empieces grandes proyectos con ideas pequeñas".

\section{REFERENCIAS BIBLIOGRÁFICAS}

Apps, Ann \& McIntire, Ross (2000), "Dublin Core Metadata for Electronic Journals", en Research and Advanced Technology for Digital Libraries. Lecture Notes in Computer Science, Volume 1923/2000, 93-102, DOI: 10.1007/3-540-45268-0_9.

Cetto, Ana María (1998), "Scientific Periodicals in Latin America and the Caribbean: A Global Perspective", en Interciencia,1998, 23:84-93.

Collier, Harry R. (1981), “Definition of 'Electronic Publishing”, en Electronic Document Delivery - II: Proceedings of a Workshop and Exhibition Organized by the Commission of the European Communities. Directorate-General Information Market and Innovation. Luxembourg, 18-19 December 1980, pp. 27-40, Edited by J.R.U. Page, Oxford: Learned Information, 1981, 214 pp.,

Collier, Harry B. (1984), "The Concept of Learned Information's Electronic Magazine”, en Electronic Publishing Review, Vol. 4, núm. 3 (1984), pp. 179-188, Oxford: Learned Information, ISSN: 0260-6658.

Dappert, Angela \& Enders, Markus (2008), "Using METS, PREMIS and MODS for Archiving eJournals", en D-Lib Magazine, September/ October 2008, Vol. 14 núm. 9/10, ISSN 1082-9873, disponible en: http://www.dlib.org/dlib/september08/dappert/09dappert.html.

IDF - International DOI Foundation (2010), “The Digital Object Identifier (DOI) System”, disponible en: http://www.doi.org/. 
McKiernan, Gerry (2000), “EJI: A Registry of Innovative E-Journal Features and Functionalities", Iowa State University, disponible noviembre 3, 2005: http://www.public.iastate.edu/ CYBERSTACKS/EJI.htm.

"Melero, Remedios (2005), "Significado del acceso abierto (open-access) a las publicaciones científicas: definición, recursos, copyright e impacto", en El profesional de la información, Vol. 14, núm. 4, pp. 255-266, disponible en: http://www.elprofesionaldelainformacion.com/contenidos/2005/julio/3.pdf.

"Mobile Web Best Practices 1.0 - Basic Guidelines, W3C Proposed Recommendation" (2006), disponible en: http://www.w3.org/ TR/2006/PR-mobile-bp-20061102/.

"New Horizons in Adult Education" (1987), Vol. 1, núm. 1, Fall, 1987, disponible en: http://www.nova.edu/ aed/horizons/vol1n1.

Okerson, Ann. (1991), "The Electronic Journal: What, Whence, and When?" en The Public-Access Computer Systems Review, Vol. 2, núm. 1, pp. 5-24, 1991.

Web Design Group (2006), "Standards for HTML Authoring for the World Wide Web”, October 2006. W3C World Wide Web Consortium, disponible en: http://www.htmlhelp.com/design/standards. html\#Validation.

W3C - World Wide Web Consortium (2011), “HTML5 Edition for Web Authors revision 1.4626: a vocabulary and associated API's for HTML and XHTML", W3C Working Draft 13 January 2011, disponible en: http://www.w3.org/TR/html5/author/.

Zeldman, Jeffrey (2006), Designing with Web Standards: Voices That Matters, 2nd Edition, Berkeley, CA: New Riders. 